\section{New products for oral care and healing}

StellaLife, an innovator in biotechnology products, has launched a new line of products for oral pain management, accelerated healing, and long-term oral healthcare. The patent-pending VEGA Oral Care system is formulated to improve dental postsurgical recovery and optimise oral systemic health. It is the first line of products launched by StellaLife.

The VEGA Oral Care system consists of three natural products: a gel, a rinse, and a recovery kit that contains a spray in addition to the gel and rinse. The VEGA products accelerate healing and reduce pain, swelling, and bruising. They also contain anxiolytic properties to help reduce anxiety associated with dental procedures. The spray is for sublingual application, the gel is a topical application, and the rinse is a mouthwash that is antimicrobial and enhances wound healing.

StellaLife VEGA Oral Care products contain 16 active ingredients that are part of Homeopathic Pharmacopeia of the United States (HPUS). The products can be used after all dental surgical procedures, including bone and tissue grafting, extractions, implant placements, osseous surgery, biopsies, and dental nonsurgical procedures such as scaling and root planing, and quadrant dentistry.

StellaLife is a biotechnology company that formulates oral health and pain management products that improve dental patients' experience. VEGA Oral Care solutions are clinically proven to accelerate healing and reduce pain, bruising and swelling after surgical and non-surgical procedures. In addition, its solutions deliver exceptional long-term oral health care. For more information, visit www.stellalifehealing.com.

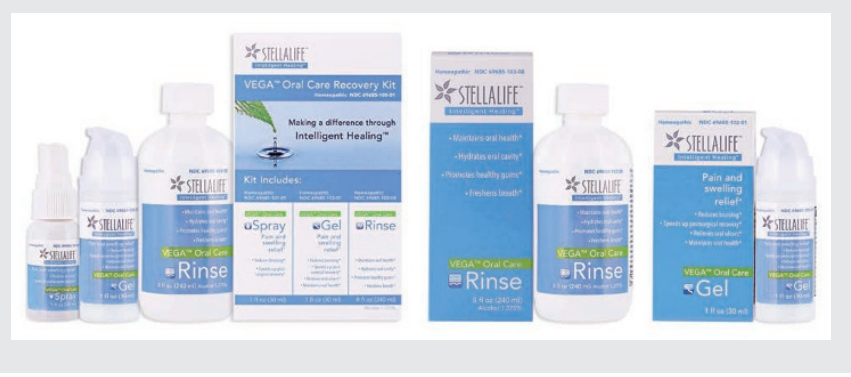

\section{Bigger and better than ever}

RPA Dental's range of products just keeps getting bigger and better. That's because the premium dental equipment distributor continues to work tirelessly in partnership with a number of quality manufacturers from around the world, adding new and innovative solutions to its catalogue all the time. Top of the range brands available from RPA Dental include Castellini, MyRay, Stern Weber and Tavom.

The first class distributor has even added a range of exquisite dental uniforms from leading Italian designer, Pastelli, to its directory.

RPA Dental's equipment sales team and Pastelli style consultants are contactable by both phone and email. Thus, if you have any questions, want a quote on a specific item or are simply looking to make an enquiry about refurbishing your practice, get in touch!

Visit www.rpadental.net. For London and Manchester Sales and Service Centres call 08000933975. 